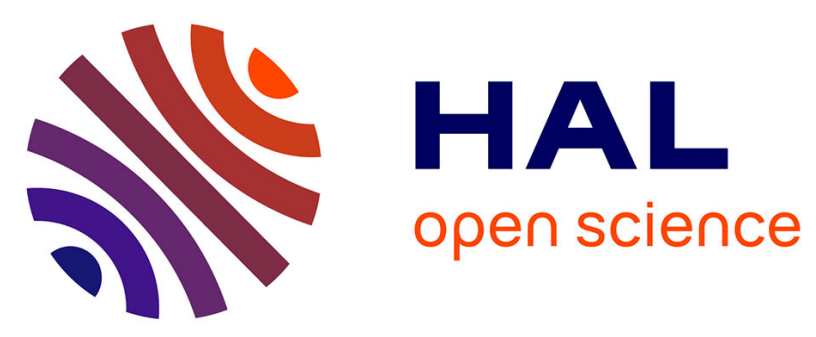

\title{
Phenomenological reduced-order modeling of subcritical combustion in cryogenic rocket engines: an application to the prediction of high-frequency combustion instabilities
}

\author{
Guillaume Storck, Luc-Henry Dorey, Sebastien Ducruix, Marie Theron
}

\section{To cite this version:}

Guillaume Storck, Luc-Henry Dorey, Sebastien Ducruix, Marie Theron. Phenomenological reducedorder modeling of subcritical combustion in cryogenic rocket engines: an application to the prediction of high-frequency combustion instabilities. AIAA Propulsion and Energy 2020 Forum, Aug 2020, Virtual event, United States. pp.AIAA 2020-3830, 10.2514/6.2020-3830 . hal-03177668

\author{
HAL Id: hal-03177668 \\ https://hal.science/hal-03177668
}

Submitted on 23 Mar 2021

HAL is a multi-disciplinary open access archive for the deposit and dissemination of scientific research documents, whether they are published or not. The documents may come from teaching and research institutions in France or abroad, or from public or private research centers.
L'archive ouverte pluridisciplinaire HAL, est destinée au dépôt et à la diffusion de documents scientifiques de niveau recherche, publiés ou non, émanant des établissements d'enseignement et de recherche français ou étrangers, des laboratoires publics ou privés. 


\title{
Phenomenological reduced-order modeling of subcritical combustion in cryogenic rocket engines: an application to the prediction of high-frequency combustion instabilities
}

\author{
Guillaume Storck* and Luc-Henry Dorey ${ }^{\dagger}$ \\ ONERA, Université Paris-Saclay, Palaiseau 91123 FRANCE \\ Sébastien Ducruix \\ Laboratoire EM2C, CNRS, CentraleSupélec, Université Paris-Saclay, Gif-sur-Yvette 91190, FRANCE \\ Marie Théron ${ }^{\S}$ \\ CNES, DLA, Paris 75012 FRANCE
}

The set of equations governing reacting two-phase flows in liquid-propellant rocket engines is simplified and linearized around a steady base flow. In the particular case of a liquid/gas coaxial injector, models for the atomization, evaporation and combustion source terms are derived from a simplified physical description of both the oxidizer liquid core and spray. The resulting equations are projected onto a modal basis, leading to a quasi-linear dynamic description of the evolution of the system. In this paper, detailed model reduction focuses on the evaporation of polydisperse sprays and on the propagation of acoustic waves within the gas.

\section{Nomenclature}

$\begin{array}{lll}\langle\cdot\rangle & = & \text { base flow quantity } \\ \cdot & = & \text { perturbation quantity } \\ \cdot & = & \text { average along a given direction } \\ \cdot g & = & \text { gas-phase quantity } \\ \cdot l & = & \text { spray quantity } \\ \cdot & = & \text { dot product in } \Omega \\ (\cdot \mid \cdot) & = & \text { inner product in } \Sigma \\ C_{l} & = & \rho_{l} / \rho_{g} \text { density ratio } \\ L_{c} & = & \text { length of the liquid core }[\mathrm{m}] \\ n & = & \text { number density function }(\mathrm{NDF})\left[\mathrm{m}^{-4}\right] \\ s & = & \text { size coordinate (diameter) }[\mathrm{m}] \\ S_{c} & = & \text { cross-sectional area of the liquid core }\left[\mathrm{m}^{2}\right]\end{array}$

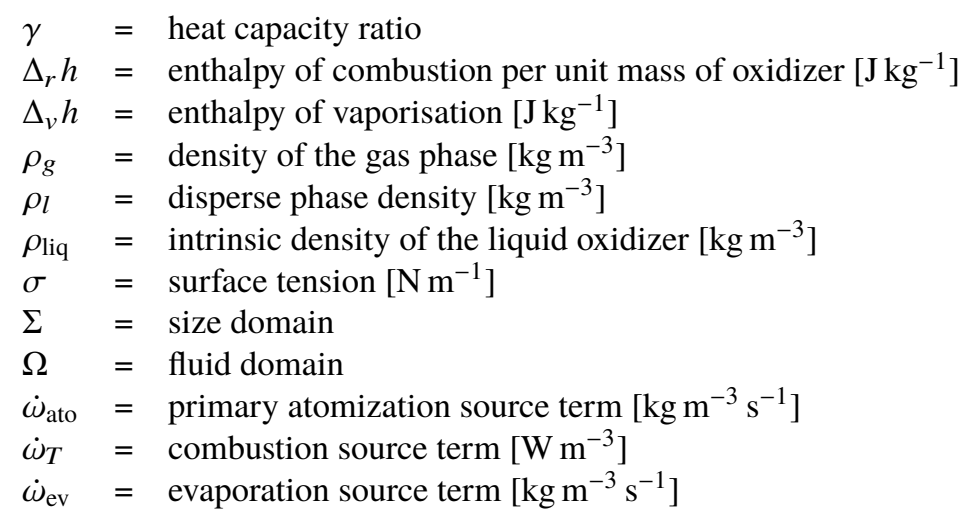

\section{Introduction}

$I^{\mathrm{N}}$ liquid-propellant rocket engines (LREs), high-frequency instabilities mainly arise from the coupling of acoustics, 1 combustion and flow dynamics within the combustion chamber (see fig. 1] [1-3]. These destructive phenomena may trigger a surge in both the chamber pressure and the total wall heat flux, putting the structural integrity of the engine at risk [4]. Moreover, even mild instabilities hamper the controllability of a rocket, which is especially problematic now that landing or throttling capabilities and precise trajectory control for multiple payloads have become desirable features. These instabilities have been observed and studied since the 1950s, yet their exact mechanism is still under scrutiny. With the recent progress in computing power, numerical tools such as reactive, multiphase Large-Eddy-Simulations

\footnotetext{
*PhD Student, DMPE/MPF, email: guillaume.storck@ onera.fr

${ }^{\dagger}$ Research Scientist, DMPE/MPF, email: luc-henry.dorey@onera.fr (corresponding author)

$\ddagger$ Director, Laboratoire EM2C

${ }^{\S}$ Combustion Devices Expert, CNES/DLA
} 
(LES) have become attractive. The latter provide a high-fidelity description of the physical processes that take place in the chamber at a relatively low cost compared to experiments, which is appreciable both for basic understanding and design validation. In fact, LES give access to quantities that are not even measurable experimentally due to the extreme conditions in the chamber [5-8]. Yet, their high cost and long run time still make them unsuitable for preliminary design phases.

Access to space is increasingly being regarded as a commodity; as competition increases, the use of design optimization tools becomes inevitable to drive the costs of space launchers further down. These tools need to simulate a large number of designs and operating points before converging to an optimum. However, designers need to ensure that these designs remain stable within their operating ranges. A full-blown LES at a cost of millions or dozens of millions of CPU hours clearly does not fit into that scheme. This is why reduced-order models (ROMs) are required to assess the stability of a given engine during the optimization process. Semi-empirical analytical models have long been the preferred tool of designers, but their predictive power is rather limited because of the abstractness of their input parameters and because of the limited number of phenomena they consider [1, 2].

Here, we present an a priori ROM based on a simplified description of some source terms in the two-phase Navier-Stokes equations in the case of a subcritical flow, i.e. when both propellants (fuel and oxidizer) have injection temperatures and pressures below their subcritical points. The scope of the study is also restricted to systems with coaxial injectors. For simplicity, it will be assumed that the fuel is gaseous and that it is injected around a core of liquid oxidizer. The modeled source terms are plugged into a linearized Euler framework and the features of the resulting system are investigated using modal projection. The work presented here is inspired by [9, 10] and aims at providing a ROM for subcritical operating points that is both more accurate and readily usable with a limited knowledge of the engine's behavior.

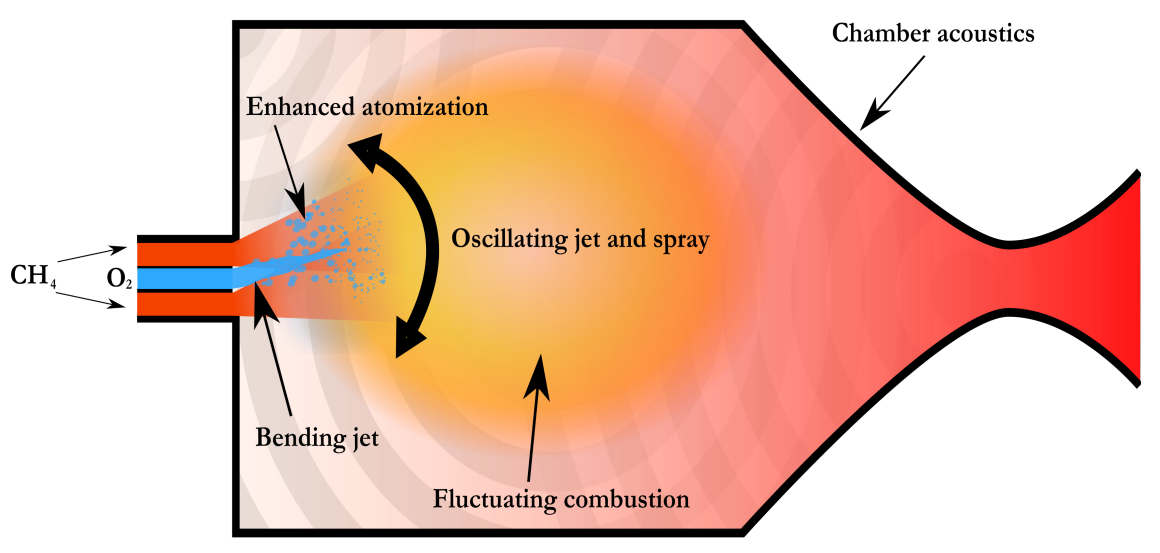

Fig. 1 In LRE, high-frequency instabilities arise from the coupling of a variety of complex phenomena, among which the dynamics of the liquid oxidizer jets and spray (in the subcritical regime). Only a single injector is depicted here for clarity, but actual LREs feature dozens to hundreds of them.

\title{
III. Governing equations
}

\author{
A. Gas phase \\ Consider a two-phase medium consisting of a gas phase and a polydispersed spray of oxygen droplets. Let us assume \\ that each phase contains only one species, and that the dispersed phase is made of incompressible particles which have
}


no influence on pressure* The equations governing the gaseous flow are the Navier-Stokes equations :

$$
\begin{cases}\partial_{t} \rho_{g}+\partial_{j}\left(\rho_{g} u_{g, j}\right) & =\dot{\omega}_{\mathrm{ev}}, \\ \partial_{t}\left(\rho_{g} u_{g i}\right)+\partial_{j}\left(\rho_{g} u_{g, i} u_{g, j}\right)+\partial_{i} p & =\nabla \cdot \sigma-\left[\partial_{t} \rho_{l} u_{l, i}+\partial_{j}\left(\rho_{l} u_{l, i} u_{l, j}\right)\right], \\ \partial_{t}\left(\rho_{g} e_{g}\right)+\partial_{j}\left(\rho_{g} e_{g} u_{j}\right)+p \partial_{j}\left(u_{g j}\right) & =\dot{\omega}_{T}-\Delta_{v} h \dot{\omega}_{\mathrm{ev}},\end{cases}
$$

where the subscripts $l$ and $g$ refer respectively to the liquid (spray) and gas phase, and $e$ designates the internal energy. Here, $\sigma$ specifically refers to the viscous stress tensor. Body forces have been neglected. The internal energy equation can be rewritten in terms of pressure using the ideal gas equation $p=\rho_{g} r T_{g}$ : in fact, for ideal gases, the internal energy depends only on temperature, with $e_{g}=\frac{r T}{\gamma-1}$. It follows that

$$
\partial_{t} p+u_{g, j} \partial_{j} p=-\gamma p \partial_{j} u_{g j}+(\gamma-1)\left[\dot{\omega}_{T}-\Delta_{v} h \dot{\omega}_{\mathrm{ev}}\right] .
$$

As a result, the system governing the evoluation of the gas phase now reads

$$
\begin{cases}\partial_{t} \rho_{g}+\partial_{j}\left(\rho_{g} u_{g, j}\right) & =\dot{\omega}_{\mathrm{ev}} \\ \partial_{t}\left(\rho_{g} u_{g i}\right)+\partial_{j}\left(\rho_{g} u_{g, i} u_{g, j}\right)+\partial_{i} p & =\nabla \cdot \sigma-\left[\partial_{t} \rho_{l} u_{l, i}+\partial_{j}\left(\rho_{l} u_{l, i} u_{l, j}\right)\right] \\ \partial_{t} p+u_{g, j} \partial_{j} p+\gamma p \partial_{j} u_{g j} & =(\gamma-1)\left[\dot{\omega}_{T}-\Delta_{v} h \dot{\omega}_{\mathrm{ev}}\right]\end{cases}
$$

\section{B. Disperse phase}

The liquid phase on the other hand obeys to the Williams-Boltzmann kinetic equation:

$$
\partial_{t} n+\nabla_{x} \cdot\left(n \mathbf{u}_{l}\right)+\nabla_{\mathbf{u}_{l}} \cdot(n \mathcal{F})+\partial_{s} n \mathcal{K}+\partial_{\theta} \mathcal{R} n=\Gamma(n)+Q(n)+A,
$$

where $n$ is the number density, such that $n\left(t, \mathbf{x}, s, \mathbf{u}_{l}, \theta\right) \mathrm{d} t \mathrm{~d} \mathbf{x} \mathrm{d} s \mathrm{~d} \mathbf{u}_{l} \mathrm{~d} \theta$ represents the number of droplets in an infinitesimal volume around $\mathbf{x}$ at time $t$ having a diameter between $s$ and $s+\mathrm{d} s$, a temperature between $\theta$ and $\theta+\mathrm{d} \theta$ and a velocity within the infinitesimal volume $\mathbf{u}_{l} \mathrm{~d} \mathbf{u}_{l}$ in the state-space. Here, $\mathcal{K}=\dot{s}_{\mathrm{ev}}$ represents the evaporation operator, $\mathcal{F}=\dot{\mathbf{u}}_{l}$ is the acceleration due to drag, $\mathcal{R}=\dot{\theta}_{\text {he }}$ symbolizes heat exchanges with the ambient gas, while the operators $\Gamma$ and $Q$ modelize fragmentation and coalescence, respectively. $A$ is the primary atomization source term.

Assuming that the distribution $n$ can be split in three factors [11], $n\left(t, \mathbf{x}, s, \mathbf{u}_{l}, \theta\right)=\tilde{n}(t, \mathbf{x}, s) n_{\theta}(\theta) n_{\mathbf{u}_{l}}\left(\mathbf{u}_{l}\right)$ and that the partial distributions in temperature and velocity are Dirac distributions, the terms related to these variables vanish. For the sake of convenience, we shall keep the same notation, so that the equation reduces to

$$
\partial_{t} n+\nabla_{x} \cdot\left(n \mathbf{u}_{l}\right)+\partial_{s} n \mathcal{K}=\Gamma(n)+A,
$$

with $n$ now a function of $(t, \mathbf{x}, s)$ only, with $\mathbf{x} \in \Omega$ and $s \in \Sigma=\left[0 ; s_{\max }\right]$. The feature of interest here is the volumic evaporation rate $\dot{\omega}_{\mathrm{ev}}$, which is obtained from

$$
\dot{\omega}_{\mathrm{ev}}=-\frac{\pi \rho_{\mathrm{liq}}}{2} \int_{\Sigma} \mathcal{K}(s) n(s) s^{2} \mathrm{~d} s,
$$

while the disperse phase density reads $\rho_{l}=\frac{\pi \rho_{\text {liq }}}{6} \int_{\Sigma} s^{3} n(s) \mathrm{d} s, \rho_{\text {liq }}$ being the density of the liquid oxidizer. An additional equation involving the velocity of the droplets $\mathbf{u}_{l}$ is required to close the system, viz.

$$
\partial_{t}\left(\rho_{l} u_{l i}\right)+\partial_{j}\left(\rho_{l} u_{l i} u_{l j}\right)=\frac{\rho_{l}}{\tau_{d}}\left(u_{g i}-u_{l i}\right),
$$

where $\tau_{d}$ is the characteristic drag time of the droplets. Under the low-Reynolds number approximation, Stokes' law can be used to compute the drag term:

$$
\tau_{d}=\frac{\rho_{\text {liq }}}{18 \mu_{g}} \int_{\Sigma} s^{2} n(s) \mathrm{d} s
$$

Using a single drag time for all droplets regardless of their size is a crude approximation, but it is made necessary by the size-velocity decoupling hypothesis made earlier. Summing up this discussion, the final system of $2 N_{d}+3$ governing

*This essentially amounts to assuming a small volume fraction of liquid. 
equations, with $N_{d}=\operatorname{dim} \Omega$, reads

$$
\begin{cases}\partial_{t} \rho_{g}+\partial_{j}\left(\rho_{g} u_{g, j}\right) & =\dot{\omega}_{\mathrm{ev}} \\ \partial_{t}\left(\rho_{g} u_{g i}\right)+\partial_{j}\left(\rho_{g} u_{g, i} u_{g, j}\right)+\partial_{i} p & =\nabla \cdot \sigma-\left[\partial_{t} \rho_{l} u_{l, i}+\partial_{j}\left(\rho_{l} u_{l, i} u_{l, j}\right)\right] \\ \partial_{t} p+u_{g, j} \partial_{j} p+\gamma p \partial_{j} u_{g j} & =(\gamma-1)\left[\dot{\omega}_{T}-\Delta_{v} h \dot{\omega}_{\mathrm{ev}}\right], \\ \partial_{t}\left(\rho_{l} u_{l i}\right)+\partial_{j}\left(\rho_{l} u_{l i} u_{l j}\right) & =\frac{\rho_{l}}{\tau_{d}}\left(u_{g i}-u_{l i}\right), \\ \partial_{t} n+\nabla_{x} \cdot\left(n \mathbf{u}_{l}\right)+\partial_{s} n \mathcal{K} & =\Gamma(n)+A .\end{cases}
$$

where the lattermost equation alone involves the size coordinate $s$, increasing the dimensionality of the problem to $N_{d}+1$.

\section{Source terms}

Atomization The atomization source term ensures the persistence of the spray which is otherwise consumed by the evaporation term. Given the small diameter of typical coaxial rocket injectors (a few millimeters) compared to the acoustic wavelengths (a few centimeters for a wave oscillating at a frequency in the range of $10 \mathrm{kHz}$ ), the liquid jets may be regarded as acoustically compact and modeled spatially as $1 \mathrm{D}$ manifolds (curved lines) oscillating in the flow. Let $\mathbf{1}_{C}$ be the indicator function of a given jet : $\mathbf{1}_{C}(t, \mathbf{x})=1$ if and only if $\mathbf{x} \in C(t)$ where $C(t)$ refers to the liquid core at time $t$. Then the atomization source term reduces to

$$
A=\frac{6 \dot{m}_{\mathrm{ox}}}{\pi \rho_{\mathrm{liq}} L_{c} \bar{S}_{c} m_{3}} \mathcal{A}(s) \mathbf{1}_{C}
$$

where $\dot{m}_{\mathrm{ox}}$ is the mass flow of oxidizer entering the domain, $L_{c}$ is the length of the intact liquid core, $\bar{S}_{c}$ is its average cross-sectional area and $\mathcal{A}(s)$ is the number distribution of the atomized droplets at time $t$, and $m_{3}$ is the third-order moment of $\mathcal{A}$. It is expected that acoustic waves alter this distribution; however, modelling this effect is a rather complex venture that goes beyond the scope of this study. In the steady state, Marmottant [12] observed that the number density of liquid fragments stemming from coaxial atomization follow a narrow normal distribution $\mathcal{N}\left(\bar{d},(0.2 \bar{d})^{2}\right)$, which can readily be restricted to a suitable interval $\Sigma=[0 ; \bar{d}+3 \sigma]$, and renormalized :

$$
A(s)=3.420\left(\frac{\dot{m}_{\mathrm{ox}}}{\rho_{\mathrm{liq}} L_{c} S_{c}}\right) \frac{1}{\bar{d}^{4}} e^{-\frac{1}{2}\left(\frac{s-\bar{d}}{0.2 \bar{d}}\right)^{2}} .
$$

The mean diameter of the fragments is derived from the most amplified wavelength of the transverse instability of the liquid core, $\lambda_{\perp}$ as detailed by Marmottant and Villermaux in [13]. Their model takes into account the operating point of the injectors, but not the direct effect of acoustics on the atomization phenomenon, as already underlined.

It has been observed that the liquid core is distorted by the flow and oscillates due to both the drag force and the acoustic radiation pressure [14]. Following a similar reasoning, one may write the momentum transport equation along the jet - which is assumed to have a constant cross-section - and obtain the system obeyed to by the velocity of the jet $\mathbf{u}_{J}$ relative to the chamber:

$$
\left\{\begin{array}{l}
\frac{\partial \mathbf{u}_{J}}{\partial t}+U_{J} \frac{\partial \mathbf{u}_{J}}{\partial l}=\frac{\mathbf{u}_{g}^{\prime}(l, y(t), t)-\mathbf{u}_{J}}{\tau_{J}}+\mathbf{a}_{\mathrm{rad}} \\
\mathbf{y}_{J}(l, t)=\int_{0}^{t} \mathbf{u}_{J}\left(t^{\prime}, l\right) \mathrm{d} t^{\prime}
\end{array}, l \in\left[0 ; L_{C}\right]\right.
$$

where $l$ is an arc length parametrization of the jet, $U_{J}$ is the bulk injection velocity of the oxidizer, $\mathbf{a}_{\text {rad }}$ designates the acceleration due to acoustic radiation and $\tau_{J}$ is the characteristic drag response time of the jet, to be modeled. This formulation can be discretized in space, albeit at a significant computational cost. Fortunately, liquid cores tend to be short compared to acoustic wavelengths in combustion chamber conditions, which allows several simplifications:

1) Neglecting their curvature, effectively reducing them to oscillating segments attached to a fixed point

2) Assuming that the acoustic velocity $\mathbf{u}_{g}^{\prime}$ is uniform along the jet: $\mathbf{u}_{g}^{\prime}\left(t, \mathbf{y}_{J}\right) \approx \overline{\mathbf{u}}_{g}^{\prime}(t)$.

Integrating eq. (12) from the origin (where $\mathbf{u}_{J}=0$ ) to the tip of the jet (where $\mathbf{u}_{J}=\mathbf{u}_{g}$ ) and dividing by the length of the jet yields a closed system governing the length-averaged movement of the jet:

$$
\left\{\begin{array}{l}
\frac{\mathrm{d} \overline{\mathbf{u}}_{J}}{\mathrm{~d} t}+\frac{\overline{\mathbf{u}}_{J}}{\tau_{J}}=\left(\frac{1}{\tau_{J}}-\frac{U_{J}}{L_{c}}\right) \overline{\mathbf{u}}_{g}^{\prime}+\overline{\mathbf{a}}_{\mathrm{rad}} \\
\overline{\mathbf{y}}_{J}(t)=\int_{0}^{t} \overline{\mathbf{u}}_{J}\left(t^{\prime}\right) \mathrm{d} t^{\prime}
\end{array}\right.
$$


Note that this short-jet approximation is not always true in rocket gas generators as well as in some academic setups such as MASCOTTE test bench at ONERA [15, 16], where atomization is less intense, resulting in rather long jets at specific operating conditions.

Evaporation We shall adopt Godsave's " $d^{2}$-law" [17]. Given the high temperature of a typical rocket combustor, the heating delay of each droplet is neglected and its temperature is assumed to be constant and equal to the saturation temperature of the oxidizer at chamber pressure $T_{\text {sat. }}$. As a result,

$$
s^{2}(t)=s_{0}^{2}-\beta t \Rightarrow \mathcal{K}=-\frac{\beta}{s},
$$

where the evaporation rate $\beta$ reads

$$
\beta=\frac{4 \lambda_{g} N u(s)}{\rho_{\text {liq }} c_{p g}} \ln \left(1+B_{T}\right)
$$

where $\lambda_{g}$ is the thermal conductivity of the ambient gas, $c_{p g}$ its heat capacity and $T_{g}$ its temperature, and $N u(s)$ is the droplet's Nusselt number, which formally depends on its size. Considering the Ranz-Marshall correlation [18] gives for instance

$$
N u(s)=2+0.6 \operatorname{Pr}_{g}^{1 / 3} \operatorname{Re}(s)^{1 / 2},
$$

which for model reduction purposes can be simplified to $N u \approx 2$ given that the evaporating droplets are expected to be small, and so is their Reynolds number. Since the droplet is supposed to have reached its saturation temperature, the Spalding transfer number $B_{T}$ [19] boils down to

$$
B_{T}=\frac{c_{p g}\left(T_{g}-T_{\text {sat }}\right)}{L_{v}}
$$

with $L_{v}$ being the latent heat of vaporization of the oxidizer. In the end, this formulation ensures that $\beta$ is effectively a constant. This simplification, however, is not without consequences, as it makes the term $\mathcal{K}$ singular at $s \rightarrow 0$; additionally, it prevents the coupling between pressure fluctuations and the latent heat of evaporation, which has been identified as a possible instability mechanism [20].

Fragmentation Droplet fragmentation through aerodynamic stresses is a crucial mechanism because it can greatly reduce the droplet size. We shall use a statistical fragmentation operator inspired by [21], i.e.

$$
\Gamma=-f_{\text {frag }}(s) n(s)+\int_{\Sigma} f_{\text {frag }}(r) \mathcal{P}(s \mid r) n(r)\left(\frac{r}{s}\right)^{3} \mathrm{~d} r
$$

where $f_{\text {frag }}$ is the characteristic fragmentation frequency - which also depends on the state of the gas, although this dependence has been omitted here for the sake of legibility - that has been modeled by Pilch and Erdman [22] as

$$
\left(\tau^{*} f_{\text {frag }}\right)^{-1}= \begin{cases}0, & W e_{s} \leq 12 \\ 6\left(W e_{s}-12\right)^{-1 / 4}, & \left.\left.W e_{s} \in\right] 12 ; 18\right] \\ 2.45\left(W e_{s}-12\right)^{1 / 4}, & \left.\left.W e_{s} \in\right] 18 ; 45\right] \\ 14.1\left(W e_{s}-12\right)^{-1 / 4}, & \left.\left.W e_{s} \in\right] 45 ; 350\right] \\ 0.766\left(W e_{s}-12\right)^{1 / 4}, & \left.\left.W e_{s} \in\right] 350 ; 2670\right] \\ 5.5, & W e_{s}>2670\end{cases}
$$

with $W e_{s}=\frac{\rho_{g} s}{\sigma}\left\|\mathbf{u}_{g}-\mathbf{u}_{l}\right\|^{2}$ the Weber number of the droplet and $\tau^{*}=\frac{s}{\left\|\mathbf{u}_{g}-\mathbf{u}_{l}\right\|} \sqrt{\frac{\rho_{\text {liq }}}{\rho_{g}}}$ being a characteristic breakup time. Note that for $W e_{s}<W e_{c r}=12$, no breakup is occurring. This expression is rather complex for model reduction purposes. Approximating this law provides a convenient surrogate:

$$
f_{\text {frag }}(s)=0.212 \sqrt{\frac{\rho_{g}}{\rho_{\text {liq }}}} \frac{\left\|\mathbf{u}_{g}-\mathbf{u}_{l}\right\|}{s} \mathbf{1}_{W e_{s}>12}
$$

with a correlation coefficient $R^{2}=0.981$ when compared to the Pilch-Erdman model for $W e_{s} \in\left[20 ; 10^{4}\right]$. From a computational perspective, this reduced formulation also has the advantage of removing the singularity at $W e_{s}=12$. 
The model $(18)$ implies that each droplet breaks up into a cloud of uniformly sized droplets. The probability that a droplet of diameter $r$ gives rise to a droplet of diameter $s<r$ is denoted $\mathcal{P}(s \mid r)$. It can be modeled in various ways; according to Hsiang and Faeth [23], in the resulting cloud, the ratio of the mass median diameter (MMD) and the Sauter mean diameter (SMD) is approximately $M M D / S M D=1.2$, where the SMD can be related to the Weber number of the parent droplet through correlations such as the one by Wert [24]. A number of probability distributions with compact support may then be used to model $\mathcal{P}(s \mid r)$; yet, because the expression of the SMD involves a Weber number, these distributions are functions of both the relative velocity and the size variable $s$, which cannot be uncoupled. As we shall see, this is an undesirable feature for model reduction purposes. In order to keep the expressions tractable, we shall consider that droplets fragment in a purely deterministic way, i.e.

$$
\mathcal{P}(s \mid r)=\delta\left(s-s_{W}(r)\right) \mathbf{1}_{W e_{r}<12},
$$

where $s_{W}(r)$ is the diameter of the secondary droplet given by Wert's correlation [24]:

$$
W e_{s}=0.32\left[W e_{r}\left(\frac{\tau_{\mathrm{frag}}}{\tau^{*}}-1.9\left(W e_{r}-12\right)^{-1 / 4}\right)\right]^{2 / 3} .
$$

Again, this piecewise model may be approximated by a much simpler form, viz.

$$
s_{W}(r)=0.4671\left(\frac{\sigma}{\rho_{g}}\right)^{1 / 4} \frac{r^{3 / 4}}{\sqrt{\left\|\mathbf{u}_{g}-\mathbf{u}_{l}\right\|}} \mathbf{1}_{W e_{r}<12} .
$$

Conversely, the diameter of the parent droplet to a droplet of diameter $s$ is given by

$$
s_{W}^{-1}(s)=2.141\left[\frac{\rho_{g}}{\sigma} u^{2} s^{4}\right]^{1 / 3}=2.141 s W e_{s}^{1 / 3} .
$$

As a result, the fragmentation operator may be recast as

$$
\Gamma(n)=-f_{\text {frag }} n(s)+9.812 W e_{s} f_{\text {frag }}\left(s_{W}^{-1}(s)\right) n\left(s_{W}^{-1}(s)\right) .
$$

Combustion Given the harsh conditions in a combustion chamber, it is reasonable to assume an infinitely fast combustion process. Thus, the oxidizer burns as soon as it evaporates and the combustion source term reads

$$
\dot{\omega}_{T}=\Delta_{r} h \dot{\omega}_{\mathrm{ev}}
$$

\section{Model reduction}

While the results derived in this section are meant to be most generic, a special focus is given on evaporation. As a consequence, the reduction of the droplet break-up and jet dynamics models will be detailed in future work.

\section{A. Disperse phase}

\section{Principle}

The system is governed by a set of $\left(2 N_{d}+3\right)$ equations and has a dimensionality of $\left(N_{d}+1\right)$, which makes its rather expensive to solve, especially if the droplet size dimension is to be accurately discretized. Cost reduction is traditionally achieved by approximating the disperse phase distribution using a collection of Dirac distributions or a piecewise constant or affine approximation [11, 21]. This turns eq. (5) into a set of $N_{s}$ equations, $N_{s}$ being the number of classes or sections considered. These local discretizations certainly are the most flexible, but their convergence rate may be slow, requiring a large number of sections to obtain an accurate description of the disperse phase. Therefore, we shall rather use a global projection of the density $n$ onto a carefully chosen Hilbert basis of the size space $\Sigma$.

First of all, note that (5) is linear with respect to $n$. The latter is a function of $(t, \mathbf{x})$ as well as $s \in \Sigma=\left[0 ; s_{\max }\right]$ with the upper bound set by the primary atomization mechanism $\left(s_{\max }=1.6 \bar{d}\right)$ since coalescence has been neglected. It is first necessary to define $L^{2}(\Sigma)=\left\{f \in \mathcal{F}(\Sigma \rightarrow \mathbb{R}) \mid\|f\|_{2}<+\infty\right\}$ where the norm $\|\cdot\|_{2}$ is the canonical $L^{2}$-norm defined as

$$
\|f\|_{2}^{2}=\int_{\Sigma} f^{2} \mathrm{~d} s .
$$




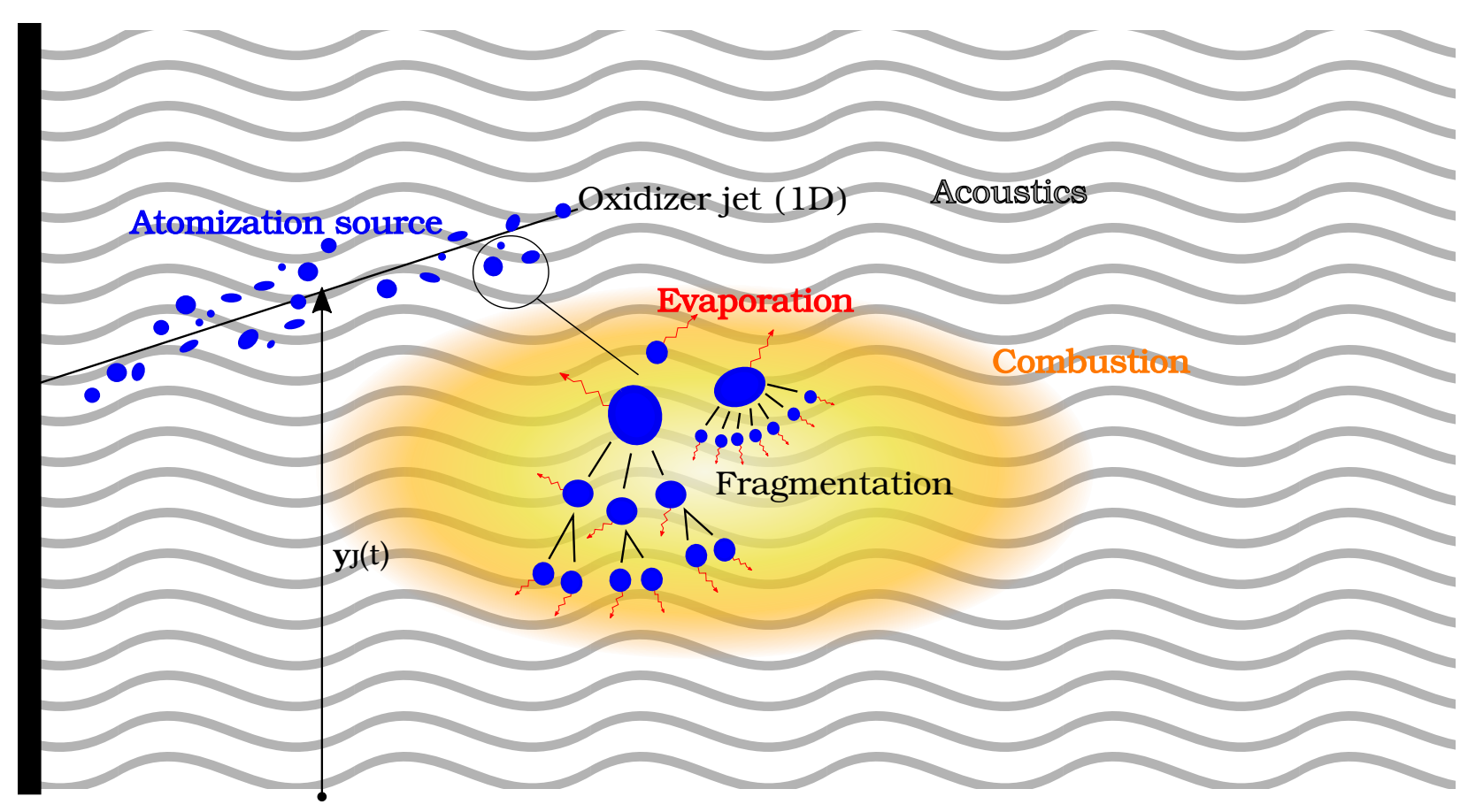

Fig. 2 The full spectrum of phenomena considered for low-order modeling covers most of the spray dynamics, as well as the time-space fluctuations of the atomization source term.

Usual modal projections use the canonical dot product in $L^{2}$ to construct a orthonormal family $\left(\pi_{i}\right)_{i \in \mathbb{N}}$ of functions of the space variable - here, the size variable $s$ - onto which the time-dependent field is to be projected. However, because of the singularity of the evaporation operator $n \mapsto \partial_{s}(n \mathcal{K})$, this functional space is not suited to our model. Therefore, we have to be careful to ensure the existence of both the modal amplitudes $\left(n \mid \pi_{i}\right)$ and the evaporation source term $\dot{\omega}_{\mathrm{ev}}$ as defined in (6). The first step is then to define the following change of variable:

$$
\varphi: s \mapsto s_{\max } e^{-z / a}, a>2, z \in[0 ;+\infty[
$$

which is a $C^{1}$-diffeomorphism. This peculiar change of variable results from multiple trials and errors; its aims are to ensure the stability of the dynamical system resulting from modal projection, as well as the existence of certain specific moments of the number density function. In the rest of this paper, $a$ is arbitrarily set to 3 . Then, if we still denote $n \equiv n \circ \varphi$ for the sake of conciseness, eq. (5) turns into

$$
\partial_{t} n+\nabla_{x} \cdot\left(n \mathbf{u}_{l}\right)+\beta^{\prime} e^{2 z / a}\left[\partial_{s} n+\frac{n}{a}\right]=\Gamma_{z}(n)+A_{z},
$$

where $\beta^{\prime}=\frac{a \beta}{s_{\max }^{2}}$ and the right-hand side terms are now expressed in terms of the $z$ variable rather than $s$. Now, define a dot product $(\cdot \mid \cdot)$ and the associated space $\mathcal{L}^{2}\left(Z=\mathbb{R}_{+}\right)$. Consider a Hilbert basis $\left(\pi_{j}\right)_{j \in \mathbb{N}}$ of $\mathcal{L}^{2}(Z)$ and let $n(t, \mathbf{x}, z)=\sum_{j=1}^{N_{s}} v_{j}(t, \mathbf{x}) \pi_{j}(z)+n^{\prime \prime}(t, \mathbf{x}, z)$. In other words, the number density can be decomposed into an $N_{s}^{\text {th }}$-order filtered approximation $\tilde{n}$ and an orthogonal residual $n^{\prime \prime}$. Introducing this decomposition into eq. (28) and taking its dot product with any of the basis functions $\pi_{i}, i \leq N_{s}$ yields

$\partial_{t} v_{i}+\nabla_{x} \cdot\left(v_{i} \mathbf{u}_{l}\right)+\left(\beta^{\prime} e^{2 z / a}\left[\frac{\pi_{j}}{a}+\frac{\mathrm{d} \pi_{j}}{\mathrm{~d} z}\right] \mid \pi_{i}\right) v_{j}-\left(\Gamma_{z}\left(\pi_{j}\right) \mid \pi_{i}\right) v_{j}=\left(A_{z} \mid \pi_{i}\right)-\left(\beta^{\prime} e^{2 z / a}\left[\frac{n^{\prime \prime}}{a}+\frac{\mathrm{d} n^{\prime \prime}}{\mathrm{d} z}\right] \mid \pi_{i}\right)+\left(\Gamma_{z}\left(n^{\prime \prime}\right) \mid \pi_{i}\right)$.

This system can be more conveniently rewritten in a matrix form; let $K_{i j}=\left(\beta^{\prime} e^{2 z / a}\left[\frac{\pi_{j}}{a}+\frac{\mathrm{d} \pi_{j}}{\mathrm{~d} z}\right] \mid \pi_{i}\right)$ and $G_{i j}=$ $\left(\Gamma_{z}\left(\pi_{j}\right) \mid \pi_{i}\right) . K^{\prime \prime}$ and $G^{\prime \prime}$ refer to the respective associated residual vectors. Finally, let $A_{i}=\left(A_{z} \mid \pi_{i}\right)$ and $N_{i}=v_{i}$. Then, eq. 29] reduces to

$$
\partial_{t} N+\nabla_{x} \cdot\left(N \mathbf{u}_{l}\right)+(K-G) N=A+G^{\prime \prime}-K^{\prime \prime} .
$$


Note that because the evaporation and fragmentation terms are nonlinear in the $z$ coordinate, it is not possible to neglect the residuals $K^{\prime \prime}$ and $G^{\prime \prime}$ in the general case. These two vectors are unclosed and require modeling, which will be addressed in section V.A.2 of this study. At this point, it is also necessary to specify which inner product shall be used; it turns out that the weighted form

$$
(\cdot \mid \cdot):(f, g) \in \mathcal{L}^{2}(Z)^{2} \mapsto \int_{0}^{+\infty} f(z) g(z) e^{-z} \mathrm{~d} z
$$

is particularly suited to this problem as it ensures that $K$ is finite when used in conjunction with the natural choice for the orthonormal basis $\left(\pi_{i}\right)_{i \in \mathbb{N}}$, i.e. the family of the Laguerre polynomials, which is orthonormal for this particular choice of dot product. In fact,

$$
K_{i j}=\beta^{\prime} \int_{0}^{+\infty} \pi_{i}\left(\frac{\pi_{j}}{a}+\frac{\mathrm{d} \pi_{j}}{\mathrm{~d} z}\right) e^{-(1-2 / a) z} \mathrm{~d} z<+\infty .
$$

Considering that the evaporation coefficient $\beta$ is a constant makes the exact computation of $K$ straightforward using a computer algebra system. The expression of $G$ on the other hand is more complex because of the strongly nonlinear dependence of $\Gamma$ on the velocity difference $\mathbf{u}_{g}-\mathbf{u}_{l}$ and the threshold effect induced by the critical Weber number condition. This term will be dealt with in future work.

\section{On the stability of the evaporation term}

It can be observed that the matrix $K$ has strictly positive eigenvalues indeed, and that it is diagonalizable. Therefore, the very simplified system

$$
\frac{\mathrm{d} N}{\mathrm{~d} t}+K N=0
$$

is stable and $\lim _{t \rightarrow+\infty} N(t)=0$. However, as $N_{s}$ increases, the eigenvalues of $N$ spread over a greater and greater part of $\mathbb{R}_{+}$, with their minimum becoming close to zero. For instance, when $N_{s}=6, \min \operatorname{Sp}(K)=6.426 \times 10^{-2} \beta^{\prime}$, whereas for $N_{s}=12, \min \operatorname{Sp}(K)=8.029 \times 10^{-3} \beta^{\prime}$. This result in a very slow relaxation of the system. Consequently, the total mass of liquid may take unphysical negative values and then increase before converging to zero. This can be avoided by choosing an appropriate, ad hoc closure model for the evaporation residual $K^{\prime \prime}$ :

$$
K^{\prime \prime}=k_{c o r r} \beta^{\prime} I_{N_{s}}
$$

where $k_{\text {corr }} \approx 1$ is a correction constant and $I_{N_{s}}$ designates the identity matrix. It should be noted that although this approach shifts all eigenvalues of $K$ to the right of the complex plane by the same amount, in practice this is only significant for the smallest of them as the order of magnitude of the shift is very small compared to most characteristic frequencies of the system. Nevertheless, this solution is far from perfect since it artificially alters the dynamics of the system as illustrated in figure 3 rather than becoming negative and then slowly going to zero - which is not physically possible - the mass of liquid decreases steadily to zero in the corrected case. Ongoing work focuses on finding a more physical closure model for the evaporation residual.

\section{B. Perturbation equations}

Consider a domain $\Omega$ having $N_{d}$ spatial dimensions and $N_{i n j}$ injectors, all of which produce acoustically compact liquid cores. The disperse phase is described by $N_{s}$ modes in the size space. Then the state vector describing the system becomes $X=\left(\rho_{g} p u_{g, i} u_{l, i} v_{j} u_{J, i}^{k}, y_{J, i}^{k}\right)^{T}, i \in \llbracket 1 ; N_{d} \rrbracket, j \in \llbracket 1 ; N_{s} \rrbracket k \in \llbracket 1 ; N_{i n j} \rrbracket$, and it obeys to the system $\lceil 9$ where the last equation has been replaced by the system (30). This is a fully nonlinear system because of the description of the gas phase. It may however be simplified using two assumptions:

1) Viscous stresses are negligible

2) The gaseous flow $X_{g}=\left(\rho_{g} p u_{g, i}\right)^{T}$ may be decomposed into a base flow $\left\langle X_{g}\right\rangle$ satisfying eq. 3 and a small perturbation $X_{g}^{\prime}$.

The base flow can be obtained from a RANS as it is a solution of the system presented here. Then the Navier-Stokes equations for the gas phase reduce to the Euler

${ }^{\dagger}$ Reynolds Averaged Navier-Stokes 


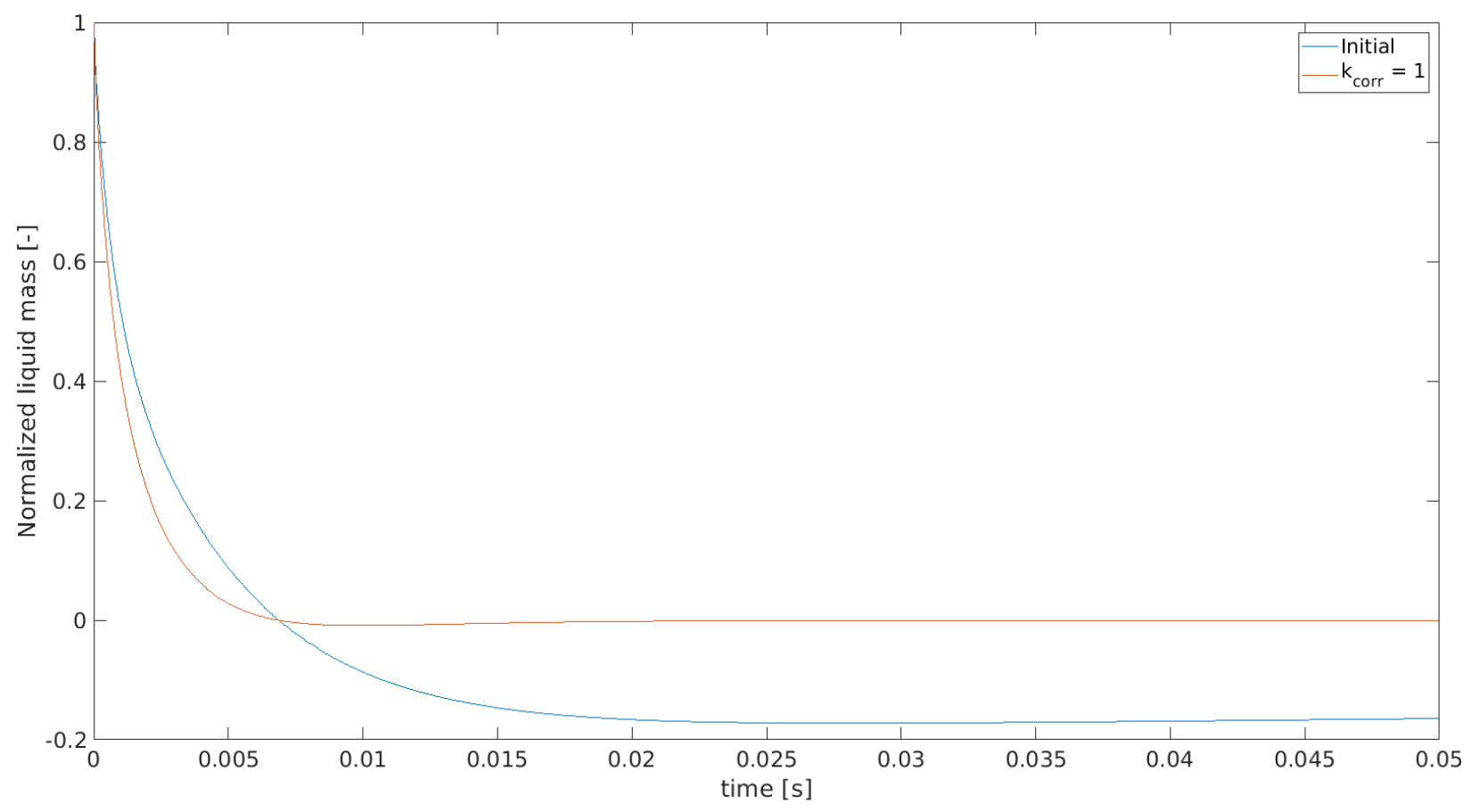

Fig. 3 Evolution of the total mass of liquid in the case presented in section VI.C, with and without the modeled unclosed term.

equations. These can subsequently be linearized around the base flow, leading to the following system:

$$
\left\{\begin{aligned}
\partial_{t} \rho_{g}^{\prime}= & \dot{\omega}_{\mathrm{ev}}^{\prime}-\left\langle\rho_{g}\right\rangle \partial_{j} u_{g j}^{\prime}-\left\langle u_{g j}\right\rangle \partial_{j} \rho_{g}^{\prime}-u_{g j}^{\prime} \partial_{j}\left\langle\rho_{g}\right\rangle-\rho_{g}^{\prime} \partial_{j}\left\langle u_{g j}\right\rangle \\
\partial_{t} p^{\prime}= & -\gamma p^{\prime} \partial_{j}\left\langle u_{g j}\right\rangle-\gamma\langle p\rangle \partial_{j} u_{g j}^{\prime}+(\gamma-1)\left[\dot{\omega}_{T}^{\prime}-\Delta_{v} h \dot{\omega}_{\mathrm{ev}}^{\prime}\right]-\left\langle u_{g j}\right\rangle \partial_{j} p^{\prime}-u_{g j}^{\prime} \partial_{j}\langle p\rangle \\
\partial_{t} u_{g i}^{\prime}= & -\left\langle u_{g j}\right\rangle \partial_{j} u_{g i}^{\prime}-u_{g j}^{\prime} \partial_{j}\left\langle u_{g i}\right\rangle-\frac{\partial_{i} p^{\prime}}{\left\langle\rho_{g}\right\rangle}+\left[\left\langle u_{l i}\right\rangle-\left\langle u_{g i}\right\rangle\right] \frac{\dot{\omega}_{\mathrm{ev}}^{\prime}}{\left\langle\rho_{g}\right\rangle}+\left(u_{l i}^{\prime}-u_{g i}^{\prime}\right) \frac{\left\langle\dot{\omega}_{\mathrm{ev}}\right\rangle}{\left\langle\rho_{g}\right\rangle} \\
& -\frac{\left\langle\rho_{l}\right\rangle}{\left\langle\rho_{g}\right\rangle}\left[\partial_{t} u_{l i}^{\prime}+\left\langle u_{l j}\right\rangle \partial_{j} u_{l i}^{\prime}\right]-\frac{\rho_{l}^{\prime}}{\left\langle\rho_{g}\right\rangle}\left\langle u_{l j}\right\rangle \partial_{j}\left\langle u_{l i}\right\rangle-\frac{\rho_{g}^{\prime}}{\left\langle\rho_{g}\right\rangle}\left\langle u_{g j}\right\rangle \partial_{j}\left\langle u_{g i}\right\rangle
\end{aligned}\right.
$$

Note that a perturbed liquid density $\rho_{l}^{\prime}$ appears in the momentum equation; this quantity can be obtained from

$$
\rho_{l}^{\prime}=\rho_{l}-\left\langle\rho_{l}\right\rangle=\frac{\pi \rho_{\text {liq }}}{6} R^{T} N-\left\langle\rho_{l}\right\rangle
$$

with $R_{k}=\frac{s_{\max }^{4}}{a} \int_{0}^{+\infty} e^{-4 z / a} \pi_{k}(z) \mathrm{d} z$. Similarly, $\dot{\omega}_{\mathrm{ev}}^{\prime}=\frac{\pi \rho_{\mathrm{liq}}}{2} L^{T} N-\left\langle\dot{\omega}_{\mathrm{ev}}\right\rangle$ with $L_{k}=\frac{\beta s_{\max }^{2}}{a} \int_{0}^{+\infty} e^{-2 z / a} \pi_{k}(z) \mathrm{d} z$. Moreover, the momentum equation of the disperse phase boils down to

$$
\partial_{t} u_{l i}^{\prime}=\frac{1}{\left\langle\tau_{d}\right\rangle}\left(u_{g i}^{\prime}-u_{l i}^{\prime}\right)
$$

Leaving the injectors aside for the sake of simplicity $\left(N_{i n j}=0\right)$, the combination of systems [30) and (35) yields

$$
\partial_{t} X+\underbrace{\left[\begin{array}{cc}
\langle A\rangle^{k} & 0 \\
0 & \left\langle u_{l, k}\right\rangle \delta_{i j}
\end{array}\right]}_{\widetilde{A}^{k}} \partial_{k} X+\underbrace{\left[\begin{array}{cc}
\langle B\rangle & \langle C\rangle \\
0 & K+\partial_{k}\left\langle u_{l, k}\right\rangle \delta_{i j}
\end{array}\right]}_{\widetilde{B}} X=\left\langle S_{C}\right\rangle+S(X)
$$


with the matrices being (if $\operatorname{dim} \Omega=3$ )

$$
\begin{aligned}
& \langle A\rangle^{k}=\left[\begin{array}{cccccccc}
\left\langle u_{g k}\right\rangle & 0 & \left\langle\rho_{g}\right\rangle \delta_{k 1} & \left\langle\rho_{g}\right\rangle \delta_{k 2} & \left\langle\rho_{g}\right\rangle \delta_{k 3} & 0 & 0 & 0 \\
0 & \left\langle u_{g k}\right\rangle & \gamma\langle p\rangle \delta_{k 1} & \gamma\langle p\rangle \delta_{k 2} & \gamma\langle p\rangle \delta_{k 3} & 0 & 0 & 0 \\
0 & \delta_{k 1}\left\langle\rho_{g}\right\rangle^{-1} & \left\langle u_{g k}\right\rangle & 0 & 0 & \left\langle C_{l}\right\rangle\left\langle u_{l k}\right\rangle & 0 & 0 \\
0 & \delta_{k 2}\left\langle\rho_{g}\right\rangle^{-1} & 0 & \left\langle u_{g k}\right\rangle & 0 & 0 & \left\langle C_{l}\right\rangle\left\langle u_{l k}\right\rangle & 0 \\
0 & \delta_{k 3}\left\langle\rho_{g}\right\rangle^{-1} & 0 & 0 & \left\langle u_{g k}\right\rangle & 0 & 0 & \left\langle C_{l}\right\rangle\left\langle u_{l k}\right\rangle \\
0 & 0 & 0 & 0 & 0 & 0 & 0 & 0 \\
0 & 0 & 0 & 0 & 0 & 0 & 0 & 0 \\
0 & 0 & 0 & 0 & 0 & 0 & 0 & 0
\end{array}\right] \\
& \langle C\rangle=\frac{\pi \rho_{\text {liq }}}{2}\left[\begin{array}{c}
-L^{T} \\
-(\gamma-1)\left(\Delta_{r} h-\Delta_{v} h\right) L^{T} \\
\frac{\left\langle u_{g}\right\rangle-\left\langle u_{l}\right\rangle}{\left\langle\rho_{g}\right\rangle} L^{T}+\frac{\left\langle u_{l, j}\right\rangle}{3\left\langle\rho_{g}\right\rangle} \partial_{j}\left\langle u_{l}\right\rangle R^{T} \\
\frac{\left\langle v_{g}\right\rangle-\left\langle v_{l}\right\rangle}{\left\langle\rho_{g}\right\rangle} L^{T}+\frac{\left\langle u_{l, j}\right\rangle}{3\left\langle\rho_{g}\right\rangle} \partial_{j}\left\langle v_{l}\right\rangle R^{T} \\
\frac{\left\langle w_{g}\right\rangle-\left\langle w_{l}\right\rangle}{\left\langle\rho_{g}\right\rangle} L^{T}+\frac{\left\langle u_{l, j}\right\rangle}{3\left\langle\rho_{g}\right\rangle} \partial_{j}\left\langle w_{l}\right\rangle R^{T} \\
0_{N_{d}}
\end{array}\right] \\
& \langle B\rangle=\left[\begin{array}{ccccc}
\nabla \cdot\left\langle\mathbf{u}_{g}\right\rangle & 0 & \partial_{x}\left\langle\rho_{g}\right\rangle & \partial_{y}\left\langle\rho_{g}\right\rangle & \partial_{z}\left\langle\rho_{g}\right\rangle \\
0 & \gamma \nabla \cdot\left\langle\mathbf{u}_{g}\right\rangle & \partial_{x}\langle p\rangle & \partial_{y}\langle p\rangle & \partial_{z}\langle p\rangle \\
0 & 0 & \partial_{x}\left\langle u_{g}\right\rangle+\frac{\left\langle C_{l}\right\rangle}{\tau_{d}}+\frac{\left\langle\omega_{\mathrm{ev}}\right\rangle}{\left\langle\rho_{g}\right\rangle} & \partial_{y}\left\langle v_{g}\right\rangle & \partial_{z}\left\langle w_{g}\right\rangle \\
0 & 0 & \partial_{x}\left\langle u_{g}\right\rangle & \partial_{y}\left\langle v_{g}\right\rangle+\frac{\left\langle C_{l}\right\rangle}{\tau_{d}}+\frac{\left\langle\dot{\omega}_{\mathrm{ev}}\right\rangle}{\left\langle\rho_{g}\right\rangle} & \partial_{z}\left\langle w_{g}\right\rangle \\
0 & 0 & \partial_{x}\left\langle u_{g}\right\rangle & \partial_{y}\left\langle v_{g}\right\rangle & \partial_{z}\left\langle w_{g}\right\rangle+\frac{\left\langle C_{l}\right\rangle}{\tau_{d}}+\frac{\left\langle\omega_{\mathrm{ev}}\right\rangle}{\left\langle\rho_{g}\right\rangle} \\
0 & 0 & -1 / \tau_{d} & 0 & 0 \\
0 & 0 & 0 & -1 / \tau_{d} & 0 \\
0 & 0 & 0 & 0 & -1 / \tau_{d}
\end{array}\right. \\
& \left.\begin{array}{ccc}
0 & 0 & 0 \\
0 & 0 & 0 \\
-\left(\frac{\left\langle C_{l}\right\rangle}{\tau_{d}}+\frac{\left\langle\dot{\omega}_{\mathrm{ev}}\right\rangle}{\left\langle\rho_{g}\right\rangle}\right) & 0 & 0 \\
0 & -\left(\frac{\left\langle C_{l}\right\rangle}{\tau_{d}}+\frac{\left\langle\dot{\mathrm{ev}}_{\mathrm{ev}}\right\rangle}{\left\langle\rho_{g}\right\rangle}\right) & 0 \\
0 & 0 & -\left(\frac{\left\langle C_{l}\right\rangle}{\tau_{d}}+\frac{\left\langle\dot{\omega}_{\mathrm{ev}}\right\rangle}{\left\langle\rho_{g}\right\rangle}\right) \\
1 / \tau_{d} & 0 & 0 \\
0 & 1 / \tau_{d} & 0 \\
0 & 0 & 1 / \tau_{d}
\end{array}\right]
\end{aligned}
$$

There are two source terms, the first of which is constant whereas the second is a nonlinear function of the state vector:

$$
\begin{aligned}
& \left\langle S_{c}\right\rangle=\left[\begin{array}{c}
-\left\langle\dot{\omega}_{\mathrm{ev}}\right\rangle \\
(\gamma-1)\left(\Delta_{r} h-\Delta_{v} h\right)\left\langle\dot{\omega}_{\mathrm{ev}}\right\rangle \\
\left(\left\langle u_{g}\right\rangle-\left\langle u_{l}\right\rangle\right) \frac{\left\langle\dot{\omega}_{\mathrm{ev}}\right\rangle}{\left\langle\rho_{g}\right\rangle}+\left\langle C_{l}\right\rangle\left\langle u_{l, j}\right\rangle \partial_{j}\left\langle u_{l}\right\rangle \\
\left(\left\langle v_{g}\right\rangle-\left\langle v_{l}\right\rangle\right) \frac{\left.\dot{\omega}_{\mathrm{ev}}\right\rangle}{\left\langle\rho_{g}\right\rangle}+\left\langle C_{l}\right\rangle\left\langle u_{l, j}\right\rangle \partial_{j}\left\langle v_{l}\right\rangle \\
\left(\left\langle w_{g}\right\rangle-\left\langle w_{l}\right\rangle\right) \frac{\left\langle\dot{\omega}_{\mathrm{ev}}\right\rangle}{\left\langle\rho_{g}\right\rangle}+\left\langle C_{l}\right\rangle\left\langle u_{l, j}\right\rangle \partial_{j}\left\langle w_{l}\right\rangle \\
0_{N_{d}+N_{s}}
\end{array}\right]
\end{aligned}
$$

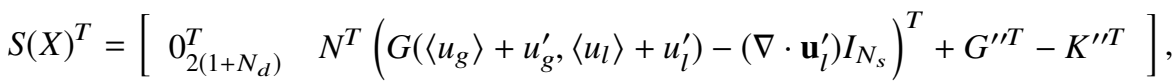


where $I_{n}$ is the identity matrix of size $n$ and $0_{n}$ is a column vector of $n$ zeros. Note that the equations of the disperse phase modal amplitudes are not linearized because of the strong nonlinearity of the fragmentation terms. In fact, this nonlinearity and the threshold effect it allows is thought to be crucial in capturing the triggering of subcritical combustion instabilities [9].

\section{Projection onto spatial modes}

Now, let $\left(\boldsymbol{\Psi}_{k}\right)_{1 \leq k \leq M}$ be an orthonormal basis of functions of $\mathbf{x} \in \mathbb{R}^{N_{d}} \rightarrow \mathbb{R}^{2\left[1+N_{d}\left(1+N_{i n j}\right)\right]+N_{s}}$, i.e.

$$
\int_{\Omega} \Psi_{i} \cdot \Psi_{j}=\delta_{i j}
$$

For each mode $k$, let $P_{k}=\operatorname{diag}\left(\Psi_{k}\right)$ be the diagonal matrix whose terms are the components of $\Psi_{k}$. Then, project the state vector $X$ onto that basis : $X(\mathbf{x}, t)=\sum_{k=1}^{M} P_{k}(\mathbf{x}) \times \mathbf{H}_{k}(t)+\mathbf{r}(\mathbf{x}, t)$ where the residual $\mathbf{r}$ and its derivatives are assumed to be orthogonal to any of the modes. This is for instance the case for empirical POD 7 modes, in which case each variable can be decomposed onto its own modal basis. The choice of the modes is not anodine as each of them must satisfy the boundary conditions imposed on the system. This is typically done by considering the acoustic eigenmodes of the cavity; however, these modes may not be optimal for the disperse phase, which is generally localized in the vicinity of the injectors, thus requiring high-frequency acoustic modes to be properly resolved. Using a specific basis for each variable alleviates this issue and allows better faster spectral convergence [25].

Introducing this decomposition into eq. $\left[38\right.$, multiplying on the left by $P_{n}, n \in \llbracket 1 ; M \rrbracket$ and integrating over the domain, one obtains

$$
\forall n \in \llbracket 1 ; M \rrbracket, \partial_{t} \mathbf{H}_{n}+\int_{\Omega} P_{n}\left(\widetilde{A}^{i} \partial_{i} P_{k}+\widetilde{B} P_{k}\right) \mathbf{H}_{k}=\int_{\Omega} P_{n}\left\langle\mathbf{S}_{c}\right\rangle+\int_{\Omega} P_{n} \mathbf{S}(X)
$$

where the summations over the $i$ and $k$ indices are implied. Denoting $E=\left(\mathbf{H}_{1}^{T} \cdots \mathbf{H}_{N}^{T}\right)^{T}, D_{n k}=\int_{\Omega} P_{n}\left(\widetilde{A}^{i} \partial_{i} P_{k}+\widetilde{B} P_{k}\right)$, $\Sigma(X)=\int_{\Omega} P_{n} S(X)$ and $\Sigma_{c}=\int_{\Omega} P_{n}\left\langle S_{c}\right\rangle$, equation 45 leads to the quasi-linear differential system

$$
\partial_{t} \mathbf{E}+D \mathbf{E}=\Sigma_{c}+\Sigma(X)
$$

It should be noted that the matrix D is of size $M^{2}\left(2+2 N_{d}\left(1+N_{i n j}\right)+N_{S}\right)^{2}$ and that it can be pre-computed as it only depends on the base flow and on the choice of a modal basis. Moreover, all the eigenvalues of $D$ should have positive (or zero) real parts as the system is stable in the absence of any source term. This final differential system may be solved using any suitable time integration scheme, i.e. any scheme that would not prevent divergence should the system be unstable. Here, we use Matlab's built-in ode15s solver [26] because of the stiffness of the $K$ matrix.

\section{Results}

In this section, we discuss the results obtained using the model in a simplified, one-dimensional case without atomization and droplet break-up. The reduced computation is compared to the output of a laminar simulation made with the CEDRE CFD platform developed at ONERA [27--29].

\section{A. Test case and numerical methods}

The purpose of this case is to highlight the salient features of the reduced-order model, especially when compared to a more complex CFD approach, and to validate its results. Consider the problem described in figure 4 it consists in a simple channel which is narrow enough to neglect acoustic effects in the transverse direction, reducing it to a 1D problem. Both boundaries are fully reflective. The base flow is quiescent and it is composed of methane at a temperature $T_{0}=1000 \mathrm{~K}$ and a pressure $p_{0}=20 \mathrm{bar}$. An initial, isentropic Gaussian pressure perturbation with a relative pressure amplitude of $1 \%$ is superimposed over this field with an offset ensuring a zero mean of $p^{\prime}(0, x)$ over the entire domain:

$$
p^{\prime}(0, x)=0.01 p_{0}\left\{\exp \left[-\frac{1}{2}\left(\frac{x-0.05}{0.0071}\right)^{2}\right]-0.0251\right\} \text {. }
$$

\footnotetext{
†Proper Orthogonal Decomposition
} 


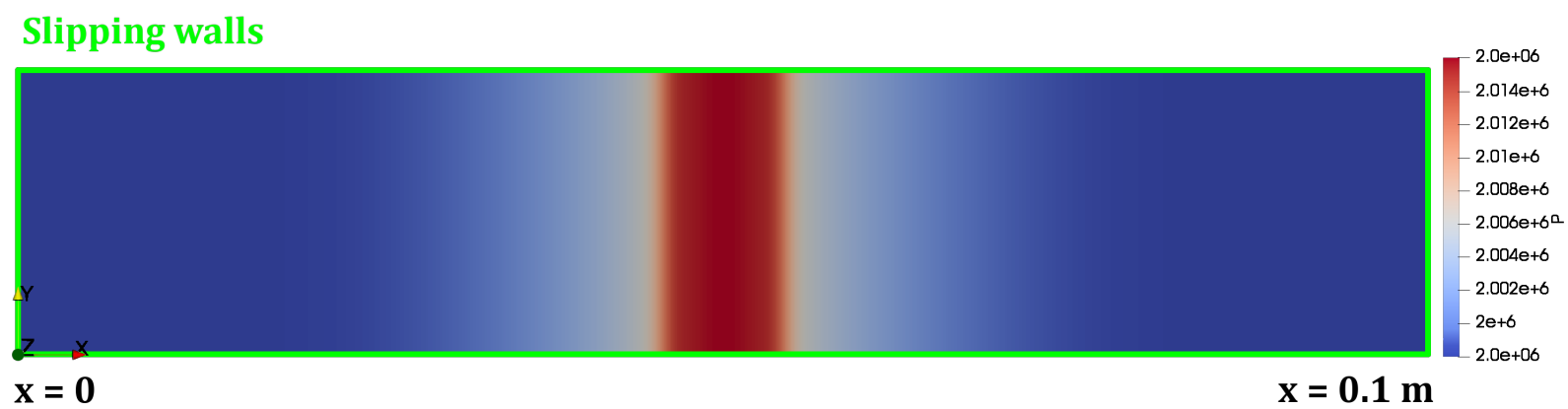

Fig. 4 Overview of the test case with the initial pressure field

The disperse phase initially has a uniform density in the domain $\Omega$ and is made of quiescent oxygen droplets following an exponential size distribution:

$$
n(s)=2.7068 \times 10^{12} \exp \left\{-\frac{s}{D_{10}}\right\}, D_{10}=20 \mu \mathrm{m}, s \in[0 ; 100] \mu \mathrm{m}
$$

Their initial temperature is $130 \mathrm{~K}$ and the total volumic fraction of liquid is $\alpha_{l}=10^{-6}$. This low value has been chosen to limit the coupling effects between the gas and the disperse phase. The method applied here has been recently used to simulate subcritical, diphasic flows, either nonreacting with coaxial air-assisted atomization [14], or reactive flows replicating experiments conducted on the MASCOTTE LRE test bench [29]. Two Eulerian solvers are used simultaneously:

- A Separated Phase Solver (SPS) called CHARME solves the compressible Navier-Stokes equations in the gas phase, here in the laminar case.

- A Disperse Phase Solver (DPS) called SPIREE solves a variant of the semi-kinetic equation (5) for the disperse phase using a sectional affine discretization of the number density distribution.

Both solvers are fully coupled through source terms. The only limitation of this approach is that the disperse phase does not alter the propagation of acoustic waves in the gaseous medium, which can be problematic in situations where $\rho_{l}$ is very large. This further motivates our choice of a very low liquid load. Tables 1 and 2 specify the setups of the CEDRE simulation and the ROM, respectively. The ROM uses ten Laguerre polynomials to reconstruct the size distribution. The initial error on the total mass of droplets between both cases is about one percent. Thus, both approaches are equivalently accurate in reconstructing the initial number density function. Moreover, each variable is projected onto a finite spatial basis of twenty Fourier modes. In this very simplified case, with a single space dimension and a quiescent base flow with no disperse phase, the constitutive terms of the system (38) boil down to

$$
\begin{aligned}
& \langle A\rangle=\left[\begin{array}{cccc}
0 & 0 & \left\langle\rho_{g}\right\rangle & 0 \\
0 & 0 & \gamma\langle p\rangle & 0 \\
0 & \left\langle\rho_{g}\right\rangle^{-1} & 0 & 0 \\
0 & 0 & 0 & 0
\end{array}\right], \\
& \langle B\rangle=\left[\begin{array}{cccc}
0 & 0 & 0 & 0 \\
0 & 0 & 0 & 0 \\
0 & 0 & 0 & 0 \\
0 & 0 & -1 / \tau_{d} & 1 / \tau_{d}
\end{array}\right], \\
& \langle C\rangle=\frac{\pi \rho_{\mathrm{liq}}}{2}\left[\begin{array}{c}
-L^{T} \\
(\gamma-1) \Delta_{v} h L^{T} \\
0_{1 \times 6} \\
0_{1 \times 6}
\end{array}\right],
\end{aligned}
$$




\section{General setup}

\begin{tabular}{ll}
\hline Solvers & DPS and SPS , two-way coupling \\
\hline Time marching & $\Delta t_{\text {DPS }}=5 \times 10^{-8} \mathrm{~s}$ \\
$\Delta t_{\mathrm{SPS}}=5 \times 10^{-8} \mathrm{~s}$
\end{tabular}

Table 1 Numerical setup of the CEDRE DPS and SPS solvers

$\left\langle S_{c}\right\rangle^{T}=\left[\begin{array}{lllllllll}0 & 0 & 0 & 0 & 0 & 0 & 0 & 0 & 0\end{array}\right]$ and $S(X)^{T}=\left[\begin{array}{lllll}0 & 0 & 0 & 0 & -\left(\nabla \cdot \mathbf{u}_{l}^{\prime}\right) I_{5} N\end{array}\right]$. In this case, the closure model (34) for the evaporation residual was used with $k_{\text {corr }}=1$, so that the matrix $K$ effectively is the corrected version of $K$, i.e. $K+k_{\text {corr }} \beta^{\prime} I$ and thus the unclosed residual $K^{\prime \prime}$ disappears from the nonlinear source term $S(X)$.

\section{B. Acoustic propagation}

Before proceeding to a full simulation, let us verify the ability of our reduced-order model to retrieve the accurate (albeit linear) propagation of acoustic waves in the cavity. To that end, we shall compare the pressure and velocity outputs of CEDRE and the ROM at $t=1 \mathrm{~ms}$ (about 8 acoustic periods) in the absence of a disperse phase $\left(\rho_{l}=0\right)$. Figure 5 loutlines the very good agreement between both codes, in terms of pressure as well as velocity. It should be emphasized, though, that the acoustic perturbation studied here has been deliberately chosen to be linear. In reality, pressure excursions in LREs may reach significant fractions of the mean chamber pressure, causing nonlinear phenomena to alter the propagation of acoustic waves. In that case, our ROM would not agree that well with the DPS. In fact, looking at the pressure history at a specific location, for instance the center of the domain, hints at the existence of slight nonlinearities. As it can be seen on figure 6 the pressure curves computed by CEDRE and the ROM are slightly out of phase; this is explained by the slight variations $\left( \pm 0.4 \mathrm{~m} \mathrm{~s}^{-1}\right)$ in the speed of sound due to the compression and expansion of the gas caused by the acoustic waves, as well as the uncertainty on the exact value of the speed of sound. This highlights the usefulness of nonlinear acoustics and accurate base flow fields when it comes to predicting the interaction between acoustics and very small features (compared to the acoustic wavelength) such as flames and jets. 


\section{General setup}

\begin{tabular}{ll}
\hline Space modes & 20 1D Fourier modes \\
\hline Time integration & Explicit variable-order stiff solver ode15s (Matlab) \\
\hline \multicolumn{1}{c}{ Gas phase } \\
\hline Base flow & $\left\langle\rho_{g}\right\rangle=3.9 \mathrm{~kg} \mathrm{~m}^{-3},\langle p\rangle=20 \mathrm{bar},\left\langle u_{g}\right\rangle=0 \mathrm{~m} \mathrm{~s}^{-1}$ \\
\hline Thermodynamics & $\gamma=1.127, \Delta_{r} h=0 \mathrm{~J} \mathrm{~kg}^{-1}$ \\
\hline Disperse phase \\
\hline Base flow & $\left\langle\rho_{l}\right\rangle=0 \mathrm{~kg} \mathrm{~m}{ }^{-3},\left\langle u_{l}\right\rangle=0 \mathrm{~m} \mathrm{~s}^{-1}$ \\
\hline Size discretization & 10 orthogonal polynomials, $s_{\text {max }}=100 \mu \mathrm{m}$ \\
\hline Physical models & $\begin{array}{l}\text { Drag time } \tau_{d}=2.8 \mathrm{~ms} \\
\text { Evaporation }\left(L_{v}=146.4 \mathrm{~kJ} \mathrm{~kg}^{-1}, \beta=1.005 \times 10^{-6} \mathrm{~m}^{2} \mathrm{~s}^{-1}\right)\end{array}$ \\
\hline
\end{tabular}

Table 2 Physical and numerical setup of the ROM
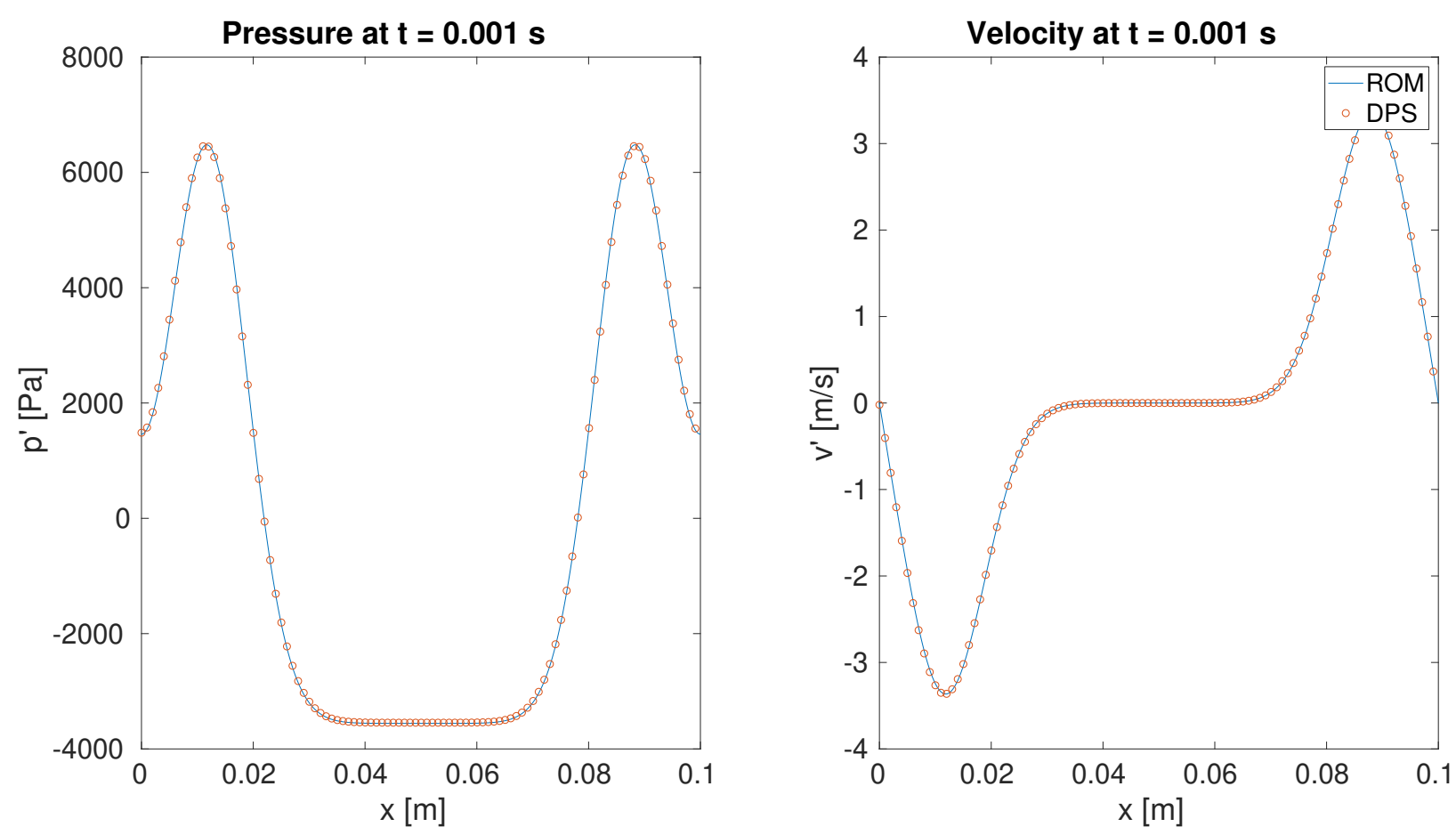

Fig. 5 Comparison of acoustic pressure (left) and velocity (right) between the ROM and CEDRE.

\section{Evolution of the disperse phase}

Now that the ability of the ROM to replicate the propagation of acoustic waves accurately enough has been verified, we shall proceed to the complete simulation as detailed in subsection VI.A Remember that the variable of interest is the 


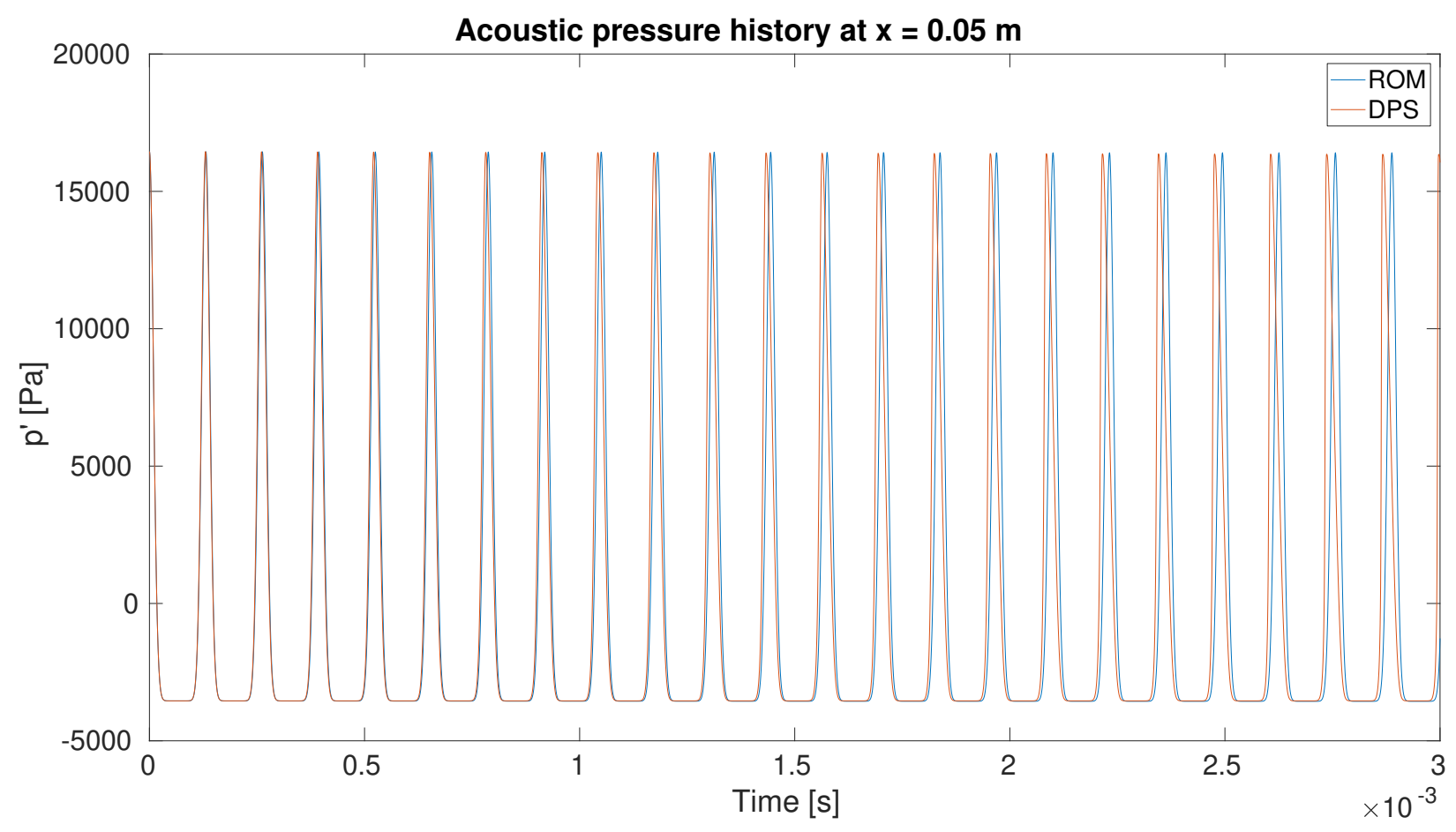

Fig. 6 Acoustic pressure history at the center of the domain for both the ROM (blue) and CEDRE (red).

evolution of the evaporation rate or, equivalently, of the density of the liquid phase. The evaporation model used in the SPS is far more advanced than the one implemented in the reduced-order model; in particular, the heating delay is not taken into account in the ROM, which can be expected to lead to a negative time lag between the output of the ROM and that of the SPS. In fact, when comparing the results given by both codes (see fig. 77, one can observe a clear similarity in the evolution of the liquid masses. However, the slopes of both curves slightly differ, leading to errors lower than $20 \%$. This discrepancy was expected, as SPS uses a more advanced evaporation model where the exchange coefficients are computed dynamically. Moreover, the ROM assumes that all droplets already are at thermal equilibrium with the ambient gas, i.e. that their temperature equals the saturation temperature of oxygen at the ambient pressure, which is $T_{\text {sat }}=132.7 \mathrm{~K}$, while the CEDRE computation is initialized with $T_{l}=130 \mathrm{~K}$ at $t=0$ out of numerical stability concerns. In addition to that, CEDRE features a more detailed description of molecular diffusion than the ROM. Yet, despite the quantitative differences in their outputs, the general agreement between both models can be regarded as acceptable and quite encouraging. Of course, the difference would be more prominent should the droplets be initialized at a much lower temperature - in that case, the CEDRE computation would have exhibited an induction period corresponding to the heating of the droplets before they reach their saturation temperature. When simulating real engines using the ROM, then, some errors should be expected in the close vicinity of the injector. In terms of computational cost, however, both codes are incomparable: the speed-up factor between CEDRE and the ROM is about 200. Of course, this is a very simple case without the highly nonlinear fragmentation term and few acoustic modes, yet it illustrates the potential of reduced-order modeling.

\section{Conclusion}

A reduced-order, quasi-linear model of subcritical diphasic flows has been derived. It is based upon simplified evolution equations for both the gaseous and disperse phase and can be updated to take into account nonlinear acoustic effects. In this paper, the solution of these equations is carried out using a Galerkin expansion of the solution over a basis of space modes, which allows to significantly decrease the computational cost of the simulation when compared to an advanced CFD tool, while providing still acceptable results. That being said, the model remains compatible with other generic PDE solution techniques, such as FEM or Discontinuous Galerkin methods. The model captures significant physical phenomena, including the time lag induced by drag as well as polydisperse evaporation, and is being updated to account for the threshold effect of droplet break-up. This allows us to believe that it may prove useful in the prediction 


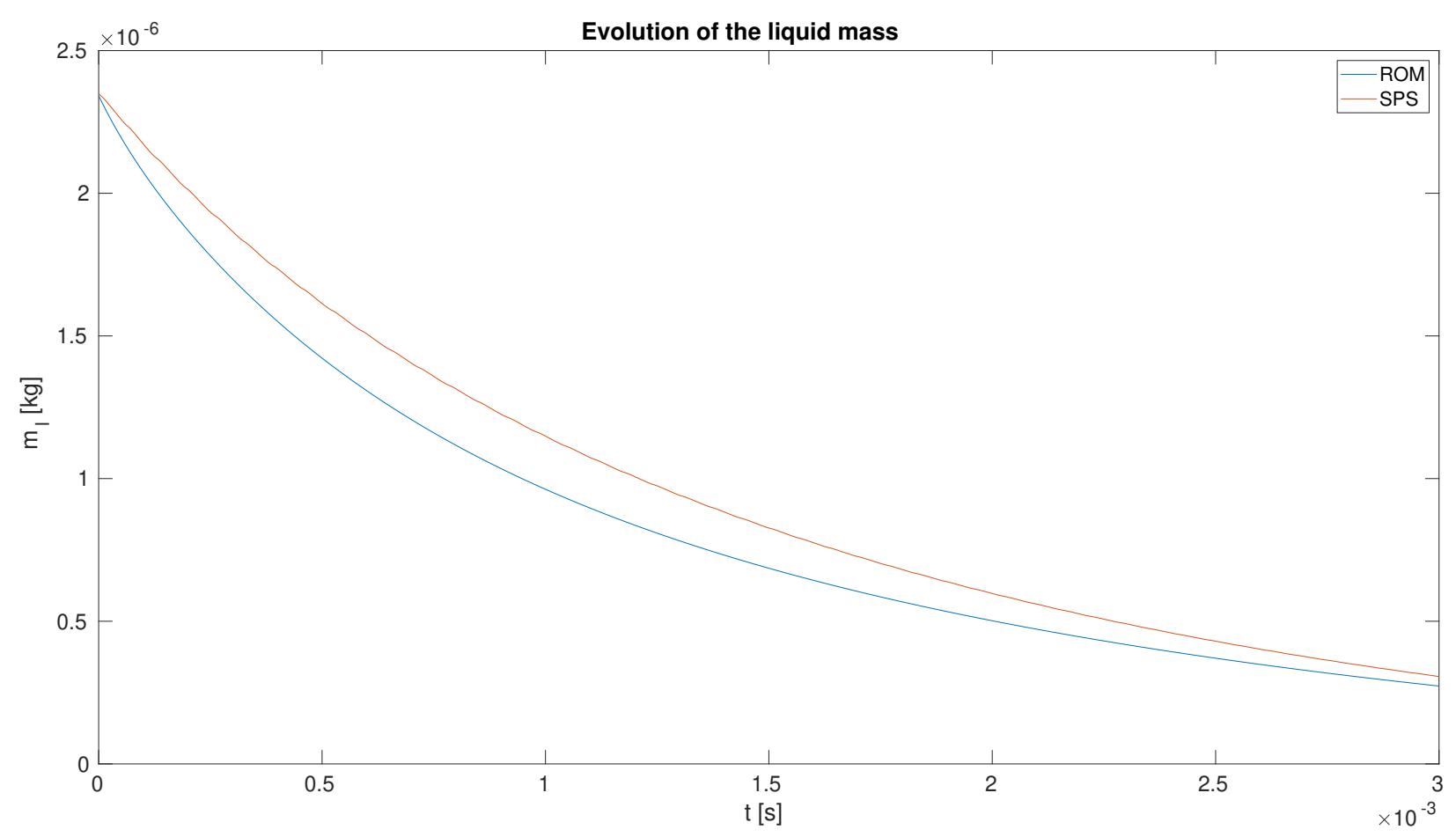

Fig. 7 Evolution of the total liquid mass within the domain with both SPS (red) and the ROM (blue).

of combustion instabilities in the subcritical regime.

Nevertheless, the ROM still requires improvement. Tests in higher dimensional cases as well as the inclusion of injectors and atomization source terms will be necessary to assess its effectiveness in more realistic cases. Following the work conducted at ONERA over the last decade [11, 14, 29], high-fidelity Large Eddy Simulations of a representative case are being carried out to confront the results of the ROM, using the CEDRE platform showcased in this study. Finally, the a priori choice of a suitable modal basis is still a current topic of research [25] and will need to be tackled.

\section{Acknowledgments}

Funding for this $\mathrm{PhD}$ work is provided by CNES, the French space agency, and ONERA.

\section{References}

[1] Anderson, W. E., and Yang, V., Liquid rocket engine combustion instability, American Institute of Aeronautics and Astronautics, 1995.

[2] Culick, F., and Kuentzmann, P., "Unsteady motions in combustion chambers for propulsion systems," Tech. rep., NATO Research and Technology Organization Neuilly-Sur-Seine (France), 2006.

[3] Brear, M. J., Nicoud, F., Talei, M., Giauque, A., and Hawkes, E. R., "Disturbance energy transport and sound production in gaseous combustion,” Journal of Fluid Mechanics, Vol. 707, 2012, pp. 53-73.

[4] Sutton, G. P., and Biblarz, O., Rocket propulsion elements, John Wiley \& Sons, 2016.

[5] Hakim, L., Schmitt, T., Ducruix, S., and Candel, S., "Dynamics of a transcritical coaxial flame under a high-frequency transverse acoustic forcing: Influence of the modulation frequency on the flame response," Combustion and Flame, Vol. 162, No. 10, 2015, pp. 3482-3502.

[6] Hakim, L., Ruiz, A., Schmitt, T., Boileau, M., Staffelbach, G., Ducruix, S., Cuenot, B., and Candel, S., "Large eddy simulations of multiple transcritical coaxial flames submitted to a high-frequency transverse acoustic modulation," Proceedings of the Combustion Institute, Vol. 35, No. 2, 2015, pp. 1461-1468. 
[7] Urbano, A., and Selle, L., "Driving and damping mechanisms for transverse combustion instabilities in liquid rocket engines," Journal of Fluid Mechanics, Vol. 820, 2017.

[8] Nez, R., Schmitt, T., and Ducruix, S., "High-frequency combustion instabilities in liquid rocket engines driven by propellants flow rate oscillations," Space Propulsion Conference, 2018.

[9] Méry, Y., "Mécanismes d'instabilités de combustion haute-fréquence et application aux moteurs-fusées," Ph.D. thesis, Châtenay-Malabry, Ecole centrale de Paris, 2010.

[10] Gonzalez-Flesca, M., Scouflaire, P., Schmitt, T., Ducruix, S., Candel, S., and Méry, Y., "Reduced Order Modeling Approach to Combustion Instabilities of Liquid Rocket Engines," AIAA Journal, 2018, pp. 1-13.

[11] Le Touze, C., "Couplage entre modèles diphasiques à «phases séparées» et à «phase dispersée» pour la simulation de l'atomisation primaire en combustion cryotechnique," Ph.D. thesis, 2015.

[12] Marmottant, P., “Atomisation d'un jet liquide par un courant gazeux,” Ph.D. thesis, Institut National Polytechnique de Grenoble-INPG, 2001.

[13] Marmottant, P., and Villermaux, E., “On spray formation,” Journal of fluid mechanics, Vol. 498, 2004, pp. $73-111$.

[14] Rutard, N., Dorey, L.-H., Le Touze, C., and Ducruix, S., "Large-eddy simulation of an air-assisted liquid jet under a high-frequency transverse acoustic forcing," International Journal of Multiphase Flow, Vol. 122, 2020, p. 103144.

[15] Vingert, L., Ordonneau, G., Fdida, N., and Grenard, P., “A rocket engine under a magnifying glass,” 2016.

[16] Méry, Y., Hakim, L., Scouflaire, P., Vingert, L., Ducruix, S., and Candel, S., "Experimental investigation of cryogenic flame dynamics under transverse acoustic modulations," Comptes Rendus Mécanique, Vol. 341, No. 1-2, 2013, pp. 100-109.

[17] Godsave, G., "Studies of the combustion of drops in a fuel spray—-the burning of single drops of fuel," Symposium (international) on combustion, Vol. 4, Elsevier, 1953, pp. 818-830.

[18] Ranz, W., Marshall, W. R., et al., "Evaporation from drops,” Chem. eng. prog, Vol. 48, No. 3, 1952, pp. $141-146$.

[19] Spalding, D., “The combustion of liquid fuels,” Proc. Combust. Inst, Vol. 4, 1953, pp. 847-864.

[20] Sohn, C., Chung, S., Kim, J., and Williams, F., "Acoustic response of droplet flames to pressure oscillations," AIAA journal, Vol. 34, No. 9, 1996, pp. 1847-1854.

[21] Dufour, G., "Modélisation multi-fluide eulérienne pour les écoulements diphasiquesa inclusions dispersées," Ph.D. thesis, PhD thesis, Université Paul Sabatier Toulouse III, 2005.

[22] Pilch, M., and Erdman, C., "Use of breakup time data and velocity history data to predict the maximum size of stable fragments for acceleration-induced breakup of a liquid drop," International journal of multiphase flow, Vol. 13, No. 6, 1987, pp. 741-757.

[23] Hsiang, L.-P., and Faeth, G. M., "Near-limit drop deformation and secondary breakup," International journal of multiphase flow, Vol. 18, No. 5, 1992, pp. 635-652.

[24] Wert, K., "A rationally-based correlation of mean fragment size for drop secondary breakup," International journal of multiphase flow, Vol. 21, No. 6, 1995, pp. 1063-1071.

[25] Laurent, C., Bauerheim, M., Poinsot, T., and Nicoud, F., "A novel modal expansion method for low-order modeling of thermoacoustic instabilities in complex geometries," Combustion and Flame, Vol. 206, 2019, pp. 334-348.

[26] Shampine, L. F., and Reichelt, M. W., "The matlab ode suite," SIAM journal on scientific computing, Vol. 18, No. 1, 1997, pp. $1-22$.

[27] Refloch, A., Courbet, B., Murrone, A., Villedieu, P., Laurent, C., Gilbank, P., Troyes, J., Tessé, L., Chaineray, G., Dargaud, J., Quémerais, E., and Vuillot, F., "CEDRE Software," AerospaceLab, , No. 2, 2011, pp. p. 1-10. URL https://hal.archives-ouvertes.fr/hal-01182463

[28] Sibra, A., Dupays, J., Murrone, A., Laurent, F., and Massot, M., "Simulation of reactive polydisperse sprays strongly coupled to unsteady flows in solid rocket motors: Efficient strategy using Eulerian Multi-Fluid methods," Journal of Computational Physics, Vol. 339, 2017, pp. 210-246.

[29] Le Touze, C., Dorey, L.-H., Rutard, N., and Murrone, A., "A compressible two-phase flow framework for Large Eddy Simulations of liquid-propellant rocket engines," Applied Mathematical Modelling, 2020. 